\title{
Experimental Study on the Compressive Strength of Big Mobility Concrete with Nondestructive Testing Method
}

\author{
Huai-Shuai Shang, ${ }^{1,2}$ Ting-Hua Yi, ${ }^{3}$ and Lu-Sheng Yang ${ }^{4}$ \\ ${ }^{1}$ School of Civil Engineering, Qingdao Technological University, Qingdao 266033, China \\ ${ }^{2}$ State Key Laboratory of Structural Analysis for Industrial Equipment, Dalian University of Technology, Dalian 116024, China \\ ${ }^{3}$ Research Center for Structural Health Monitoring and Control, School of Civil Engineering, Dalian University of Technology, \\ Dalian 116023, China \\ ${ }^{4}$ Weifang Municipal Engineering Construction Department, Weifang 261031, China
}

Correspondence should be addressed to Ting-Hua Yi, yth@dlut.edu.cn

Received 12 July 2012; Accepted 14 October 2012

Academic Editor: Rabah Khenata

Copyright ( 2012 Huai-Shuai Shang et al. This is an open access article distributed under the Creative Commons Attribution License, which permits unrestricted use, distribution, and reproduction in any medium, provided the original work is properly cited.

\begin{abstract}
An experimental study of C20, C25, C30, C40, and C50 big mobility concrete cubes that came from laboratory and construction site was completed. Nondestructive testing (NDT) was carried out using impact rebound hammer (IRH) techniques to establish a correlation between the compressive strengths and the rebound number. The local curve for measuring strength of the regression method is set up and its superiority is proved. The rebound method presented is simple, quick, and reliable and covers wide ranges of concrete strengths. The rebound method can be easily applied to concrete specimens as well as existing concrete structures. The final results were compared with previous ones from the literature and also with actual results obtained from samples extracted from existing structures.
\end{abstract}

\section{Introduction}

The direct determination of the strength of concrete implies that concrete specimens must be loaded to failure. Therefore, the determination of concrete strength requires special specimens to be taken, shipped, and tested at laboratories. This procedure may result in the actual strength of concrete, but may cause trouble and delay in evaluating existing structures. Because of that, special techniques have been developed in which attempts were made to measure some concrete properties other than strength, and then relate them to strength, durability, or any other property. Some of these properties are hardness, rebound number, resistance to penetration or projectiles, resonance frequency, and ability to allow ultrasonic pulses to propagate through concrete. However, the term "nondestructive" [1-3] is given to any test that does not damage or affect the structural behavior of the elements and also leaves the structure in an acceptable condition for the client. However, a successful nondestructive test is the one that can be applied to concrete structures in the field and be portable and easily operated with the least amount of cost.

Among the available nondestructive methods, the rebound hammer is the most commonly used one in practice. The rebound hammer test is described in ASTM C805 [4] and BS 1881: Part 202 [5]. The test is classified as a hardness test and is based on the principle that the rebound of an elastic mass depends on the hardness of the surface against which the mass impinges. The energy absorbed by the concrete is related to its strength [6]. Despite its apparent simplicity, the rebound hammer test involves complex problems of impact and the associated stress-wave propagation.

There is no unique relation between hardness and strength of concrete, but experimental data relationships can be obtained from a given concrete. However, this relationship is dependent upon factors affecting the concrete surface such as degree of saturation, carbonation, temperature, surface preparation and location, and type of surface finish [7]. The result is also affected by type of aggregate, mix proportions, 
TABLE 1: The mix proportion of big mobility concrete in per cubic meter.

\begin{tabular}{lcccc}
\hline & $\begin{array}{c}\text { Cement } \\
\left(\mathrm{kg} / \mathrm{m}^{3}\right)\end{array}$ & $\begin{array}{c}\text { Sand } \\
\left(\mathrm{kg} / \mathrm{m}^{3}\right)\end{array}$ & $\begin{array}{c}\text { Fly ash } \\
\left(\mathrm{kg} / \mathrm{m}^{3}\right)\end{array}$ & $\begin{array}{c}\text { Water } \\
\left(\mathrm{kg} / \mathrm{m}^{3}\right)\end{array}$ \\
\hline C15 & 220 & 642 & 110 & $180 \sim 190$ \\
C20 & 290 & 615 & 80 & $180 \sim 190$ \\
C30 & 390 & 587 & 70 & $180 \sim 190$ \\
C40 & 410 & 568 & 70 & $170 \sim 180$ \\
C50 & 480 & 520 & 60 & $170 \sim 180$ \\
\hline
\end{tabular}

and hammer inclination. Areas exhibiting honeycombing, scaling, rough texture, or high porosity must be avoided. Concrete must be approximately of the same age, moisture conditions, and same degree of carbonation (note that carbonated surfaces yield higher rebound values). It is clear then that the rebound number reflects only the surface of concrete. Due to the difficulty of acquiring the appropriate correlation data in a given instant, the rebound hammer is most useful for rapidly surveying large areas of similar types of concrete in the construction being considered. Neville [8] presented the benefits of using the rebound hammer in concrete and stated that the test all alone is not a strength test and the exaggerated claims of its use as a replacement for compression test should not be accepted.

A recent development in the concrete industry has been to use fly ash and powdered limestone as partial replacements for Portland cement in the production of pumped concrete. This new concrete has been widely used in China for building, bridges, and marine structures. Compressive strengthcost analysis revealed that the concrete producer could realize important saving silica fume in the concrete mixture.

In this work, the author used the rebound hammer in order to arrive at a suitable, reliable simple chart for strength evaluation of big mobility concrete. This paper presents an experimental investigation of the case of rebound hammer techniques for use as a part of C15, C20, C30, C40, and C50 big mobility concrete strength according to the Standard for Test Method of Mechanical Properties on Ordinary Concrete, GBT50081-2002 [9], and Technical Specification for Inspection of Concrete Compressive Strength by Rebound Method, JGJ/T 23-2001 [10].

\section{Experimental Program}

2.1. Materials and Mix Proportions. In this investigation, all samples were made from local materials, which consisted of the following: Chinese standard (GB175-2007) [11] Portland cement was used. Fine aggregates were natural river sand (fineness modulus of 2.6) and coarse aggregate of local natural sources or crushed hard limestone (diameter ranging from $5 \mathrm{~mm}$ to $20 \mathrm{~mm}$ ) was used. The mix proportions and the major parameters listed in Table 1 were to be adopted.

2.2. Test Specimens and Testing Programs. Five sets of C20, C25, C30, C40, and C50 big mobility concrete cubes

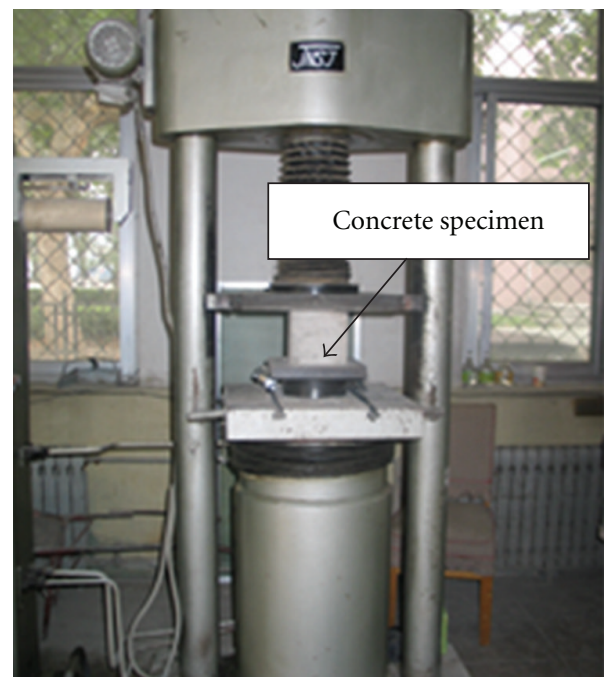

FIGURE 1: The concrete specimen on the testing machine.

$(150 \mathrm{~mm} \times 150 \mathrm{~mm} \times 150 \mathrm{~mm})$ were prepared. Each set consisted of 21 specimens. The specimens were cast in steel molds and compacted through external vibration and demoulded $24 \mathrm{~h}$ later. All the specimens were cured in a condition of $20 \pm 3^{\circ} \mathrm{C}$ and 95 percent $\mathrm{RH}$ for 27 days.

The test method starts by the careful selection and preparation of the concrete surface to be tested. Once the surface is chosen, it should be prepared by an abrasive stone so that the test surface is ground smooth. Then, a fixed amount of energy is applied by pushing the hammer against the test surface. The plunger must be allowed to strike perpendicularly to the surface. The angle of inclination of the hammer affects the result. After impact, the rebound number should be recorded. At least 16 readings must be taken from each tested area according to the JGJ/T 23-2001. Figure 1 gives the concrete specimen on the testing machine.

2.3. Research Program. The actual conditions of the sites show high variations of the materials received. These include variations in concrete quality and in the quality of workmanship, lack of technology in some cases, incorrect volume measurements of the quantities used in mixes, discontinuous supervision, and incorrect methods of concrete production usually ending in low to medium degree of quality control [12]. Therefore, it was necessary to design and follow a research program that does not depend on the previous history of the tested specimen.

The aim of the research was to obtain a simple rebound curve between rebound number through concrete and the compressive strength of big mobility concrete. The expression of the rebound curve should be as simple as possible in order to be easily used by engineers who work on-site. Also, the chart was used later for strength evaluation of some samples of concrete. The procedure that was followed during experiments consisted of the following steps. 
(1) Various concrete mixes were used to prepare standard cubes of $150 \mathrm{~mm}$ side length.

(2) Concrete cubes made under site conditions were brought from various sites for testing.

(3) Each of the two opposite faces of the cube was prepared for the rebound hammer test.

(4) The cubes were positioned in the testing machine and a slight load $(30 \sim 80 \mathrm{KN})$ was applied. The rebound number was obtained by taking measurements on the two faces of the cube. The rebound hammer was horizontal in all measurements. The results of the rebound number test were evaluated according to the rules of the JGJ/T 23-2001.

(5) Once nondestructive testing on each cube was completed, the cube was loaded to failure and the maximum load was recorded.

(6) Results were plotted as shown in Figures 2 and 3. New samples were obtained and were tested in the same way in order to check the results obtained from the curve.

(7) Six samples were taken from structures, equivalent cube strength for each sample was obtained, and the results were plotted in Table 3.

\section{Results and Discussions}

Calibration curves for each rebound method are drawn using regression analysis. The effect of degree of carbonation was represented by plotting the averages of rebound number against the compressive strength. Table 2 gives the different regress model of rebound curve between rebound number through big mobility concrete and the compressive strength of big mobility concrete according to the experimental data.

3.1. Graphical Presentation. As shown in Table 2, the bestfit curve, which represents the relationship between the rebound number and the compressive strength of concrete, is a curve which has the following equation:

$$
f_{c u}^{c}=0.032509 \times R_{m}^{1.94172} \times 10^{-0.00789 \times d_{m}},
$$

where $f_{c u}^{c}$ is the compressive strength, $R_{m}$ is the rebound number, and $d_{m}$ is the depth of carbonation. The correlative coefficient value was found to be 0.852 . The relative standard error was found to be $\mathrm{SE}=13.75 \%$.

3.2. Discussion. It is obvious that the IRH best-fit curve showed better correlation. The obtained regression model using the IRH is more accurate and gives closer results to the experimental ones than the results obtained from JGJ/T 232001.

\section{Engineering Example}

Marketplace A is concrete frame structures, and the designed compressive strength of concrete in the column is C30.

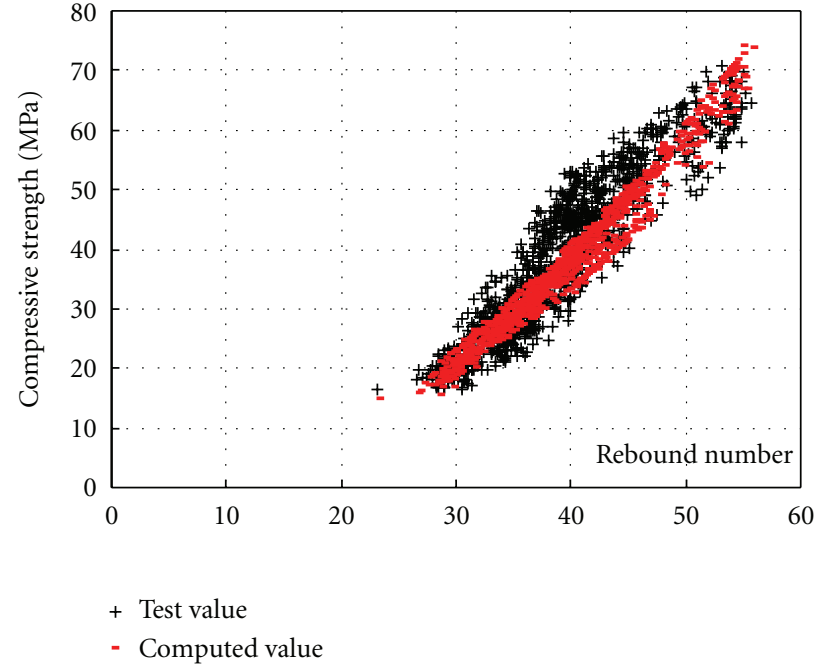

Figure 2: Test value and computed value of single-mixed big mobility concrete with rebound method.

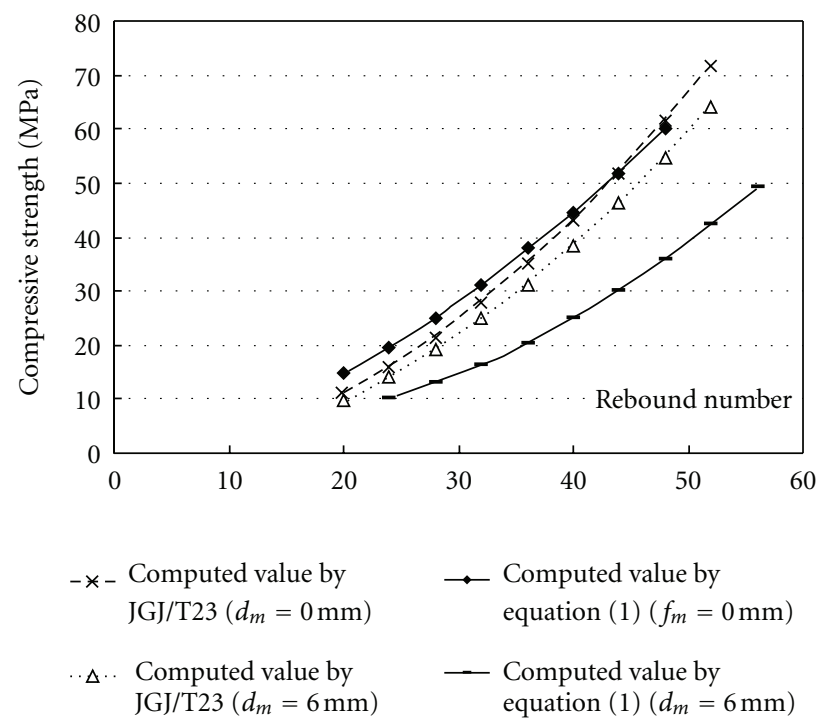

FIGURE 3: Comparison of computed value between JGJ/T23 and (1).

Date of casting is 08-04-2006, date of testing with rebound method is 22-12-2006, and date of testing with core drilling method is 23-12-2006. The application of the method to 6 samples taken from existing structures is presented in Table 3. Table 3 also shows comparison of results obtained using the following: (a) the use of (1), (b) the use of the plot given in DBJ14-026-2004 [12], and (c) the crushing compressive strength of concrete. It is clear from Table 3 that the predicted values are close to those of the observed crushing compressive strength (after adjusting the values to estimate cube strength).

The deviation between actual results and predicted results may be attributed to the fact that samples from existing structures are cores and the crushing compressive 


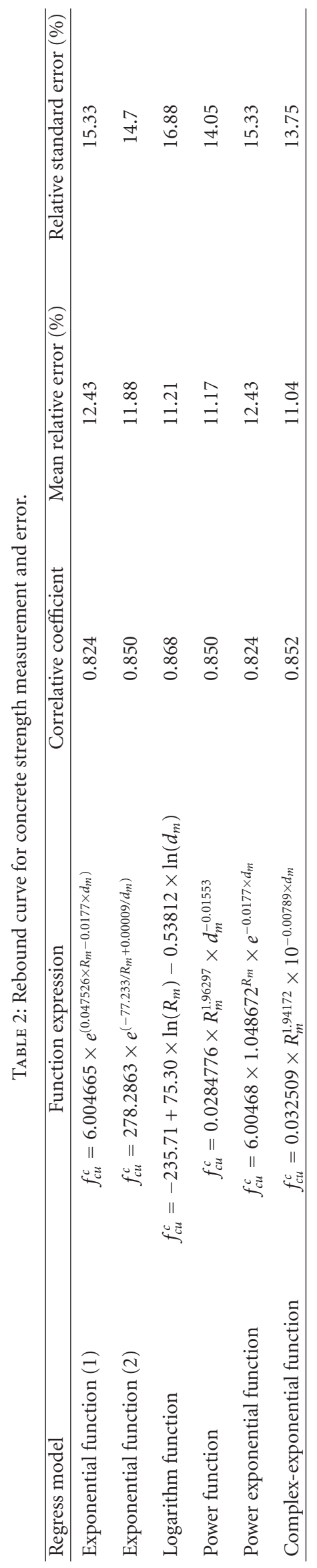




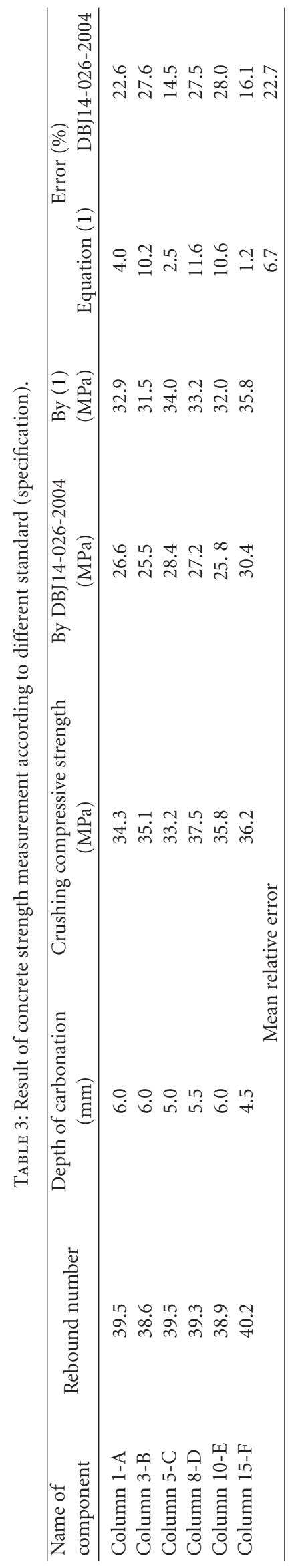


cube strength was obtained by using various corrections introduced in the specifications.

\section{Conclusion}

The development of curves to adapt rebound testing techniques for big mobility concrete mixes required revealed the following points.

(1) The use of rebound hammer is suitable to estimate and predict the strength of big mobility concrete, which makes engineering judgment quite easy. The use of the rebound hammer methods yields more reliable and closer results to the actual strength.

(2) There is no need to know the water/cement ratio of concrete since, for a specific concrete, each water/cement value yields only one value of strength which is associated with only one value of rebound number.

(3) The rebound number method seems to be more efficient in predicting the strength of concrete under certain conditions, and even that. The use of the impact rebound hammer for strength estimation of in situ concrete must never be attempted unless a specific calibration chart is available, and then, the use of this method alone is recommended.

(4) The use of rebound number method produces results that are reliable and close to the true values. In addition, an acceptable level of accuracy was achieved for strength estimation of concrete. Hence, the resulting regression model for strength evaluation could be used safely for concrete strength estimation for the concrete engineering investigation.

(5) Better results of prediction of strength are obtained for estimated crushing cube strengths. The method can be extended to test existing structures by taking direct measurements on concrete elements.

\section{Acknowledgments}

This research work was jointly supported by the Science Fund for Creative Research Groups of the National Natural Science Foundation of China (Grant no. 51121005), the National Natural Science Foundation of China (Grants no. 51208273, 51222806), a Project of Shandong Province Higher Educational Science and Technology Program (Grant no. J12LG07), and the Program for New Century Excellent Talents in University (Grant no. NCET-10-0287).

\section{References}

[1] M. Colombo and R. Felicetti, "New NDT techniques for the assessment of fire-damaged concrete structures," Fire Safety Journal, vol. 42, no. 6-7, pp. 461-472, 2007.

[2] B. Hobbs and M. Tchoketch Kebir, "Non-destructive testing techniques for the forensic engineering investigation of reinforced concrete buildings," Forensic Science International, vol. 167, no. 2-3, pp. 167-172, 2007.
[3] A. M. Mahmoud, H. H. Ammar, O. M. Mukdadi et al., "Nondestructive ultrasonic evaluation of CFRPconcrete specimens subjected to accelerated aging conditions," NDT and E International, vol. 43, no. 7, pp. 635-641, 2010.

[4] ASTM C 805-85, Test for Rebound Number of Hardened Concrete, ASTM, USA, 1993.

[5] BS 1881: Part 202, 1986: Recommendations for Surface Hardness Tests by the Rebound Hammer, BSI, UK, 1986.

[6] In Place Methods for Determination of Strength of Concrete; ACI Manual of Concrete Practice-part 2: Construction Practices and Inspection Pavements, ACI 228.1R-989, Detroit, Mich, USA, 1994.

[7] S. Amasaki, "Estimation of strength of concrete structures by the rebound hammer," CAJ Proceding of Cement and Concrete, vol. 45, pp. 345-351, 1991.

[8] A. Neville, Properties of Concrete, Addison-Wesley Longman, 1995.

[9] GBT50081-2002. National Standard of the People's Republic of China. Standard for test method of mechanical properties on ordinary concrete, 2002.

[10] JGJ/T 23-2001. National Standard of the People's Republic of China. Technical specification for Inspection of Concrete Compressive Strength by Rebound Method, 2002.

[11] National Standard of People's Republic of China, "Common Portland Cement; GB175-2007," Standards Press of China, Beijing, China, 2007.

[12] H. Qasrawi, "Quality control of concrete at site," Civil Engineering Journal, pp. 1-4, 1994. 

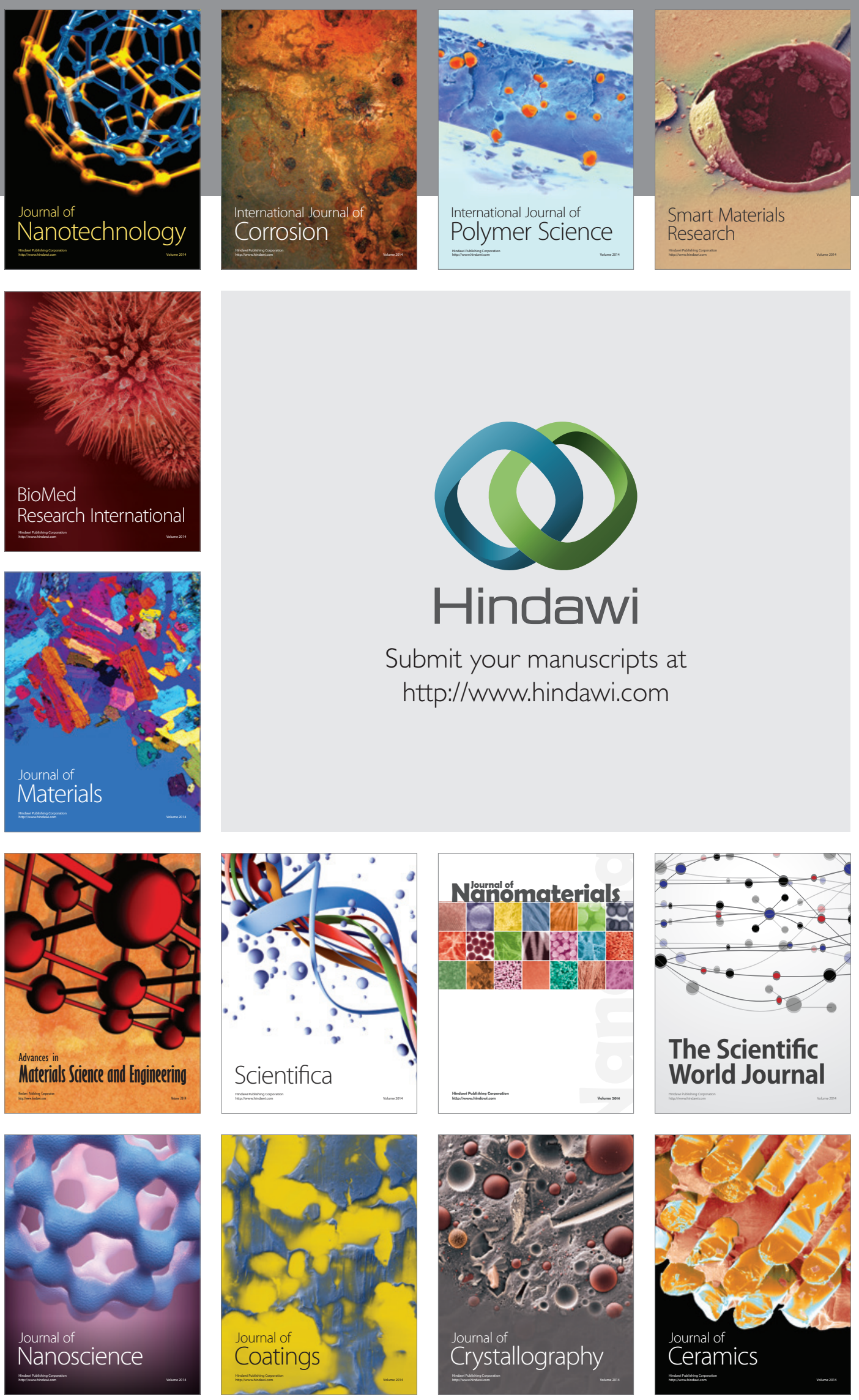

The Scientific World Journal

Submit your manuscripts at

http://www.hindawi.com

\section{World Journal}

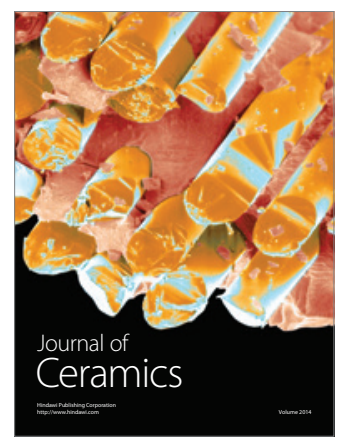

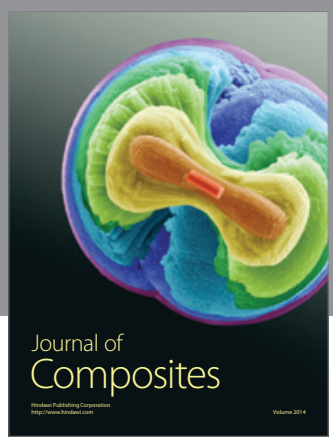
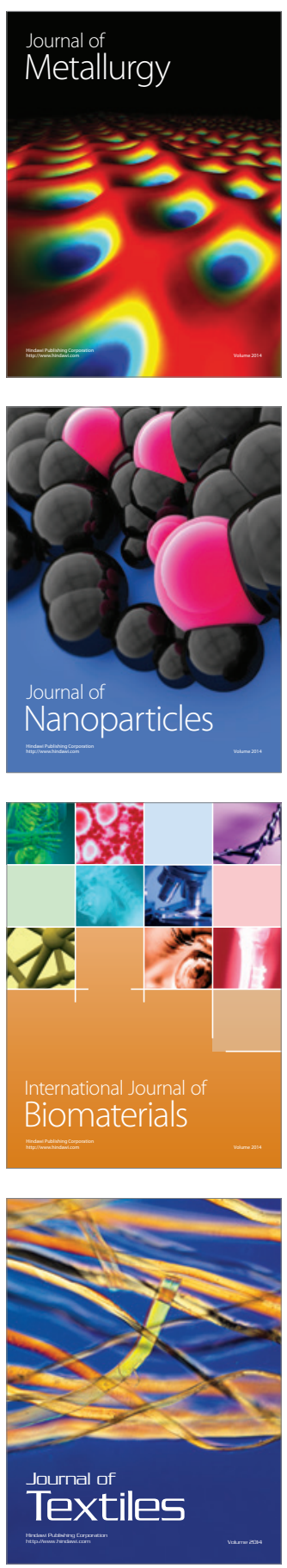